\title{
Immune System of Primary School-Age Children With Functional Respiratory Disorders in the Conditions of Anthropogenic Influence
}

\author{
Alikina I.N.* Dolgikh O.V. \\ Federal Scientific Center for Medical and Preventive Health Risk Management Technologies, Perm, Russia \\ *Corresponding author. Email: 27konti72@mail.ru
}

\begin{abstract}
The study involved 55 children with respiratory disorders aged 6 to 11 years living in the area of influence of exogenous chemical factors. The identification of the marker of cell differentiation CD127-, the level of expression of the Bcl-2 protein, the TNFR receptor was carried out by flow cytometry. Phagocytic activity of cells was studied using formalinized sheep erythrocytes. The IgG content was determined by the method of radial immunodiffusion. The level of beta2-microglobulin was determined by enzyme-linked immunosorbent assay. The functional state of the respiratory system was assessed by spirometry and rhinomanometry methods. Functional reserves were determined by calculating the Skibinskaya index. The prevalence of allergic diseases in the examined children was higher. The allergic rhinitis was diagnosed more often rather than in the comparison group. The prevalence of bronchial asthma, clinical signs of chronic lymphoproliferative diseases significantly exceeded the level of children in the comparison group. Changes in immune reactivity were established relative to the comparison group manifested in an increase in the percentage of phagocytic cells, beta2-microglobulin protein, IgG, CD127-, TNFR, and specific IgG to aluminum. Inhibition of $\mathrm{Bcl}-2$ protein expression was recorded $(\mathrm{p}<0.05)$. In children living in the area of influence of exogenous chemical factors, more often than in children of the comparison group, respiratory diseases with an allergic component were diagnosed, reliably associated with the contamination of biological media with aluminum compounds. Immunological examination revealed an imbalance between the humoral and cellular links of immunity: overproduction of specific IgG to aluminum, overexpression of proteins and receptors - beta2-microglobulin, IgG, CD127-, TNFR and Bcl-2 deficiency, which reflects the state of cellular immunodeficiency and the likelihood of allergic manifestations.
\end{abstract}

Keywords: respiratory system, immune system, contamination, aluminum, chemical risk factors, children

\section{INTRODUCTION}

The growing anthropogenic pollution of the environment has a pronounced negative impact on human health [1]. Vulnerable groups are pregnant women and children [2]. The highest risk to the health of children is observed in large industrial centers with predominantly metallurgical and chemical industries, where the body of residents is exposed to the combined effect of the components of industrial emissions and an increased content of salts in drinking water, which is the main factor in the development of pathology of various organs and systems $[3,4]$.

The immune system, which is one of the key regulatory systems, is highly sensitive to environmental changes and interactions with chemicals. Chemical reagents have a direct and indirect immunotoxic effect, acting on immunocompetent cells, changing their metabolism and exerting a mutagenic effect, modifying the biomolecules of its own body, converting them into antigens (foreign molecules). In the aggregate, negative environmental factors affect the human immune system, as a result of this the states of immunodeficiency of both specific and nonspecific defense mechanisms develop. A decrease in the body's defenses directly or indirectly contributes to the development of respiratory diseases [5-7]. Air pollution makes a significant contribution to the level of chronic somatic diseases, especially respiratory diseases $[8,9]$. Combining the harshness of the weather conditions with a high level of air pollution the pathological process often occurs in the form of acute colds or other respiratory diseases such as stenosing laryngotracheitis, obstructive bronchitis followed by chronicity of processes and the development of bronchial asthma [10].

Currently, the problem of preserving and strengthening the health of the population living in various environmental conditions is being the highest priority in the development of medicine and society. 
The purpose of this study is to assess the immune status of primary-age children with functional disorders of the respiratory system living in an area characterized by aerogenic exposure to aluminum.

\section{MATERIALS AND METHODS}

To identify the connection between destruction of the somatic health among children with unfavorable sanitary and hygienic conditions in the territory of residence, a comparative study of indicators of the clinical and functional state of life support systems and the frequency of registration of pathology by priority organs (target systems) with an assessment of immunoregulation in children attending school was carried out. 93 children were examined 55 of whom are children living in the area of influence of aluminum production enterprises, having functional disorders of the respiratory system. The comparison group consisted of children $(n=38)$ who did not have complaints and functional deviations from the respiratory system. The gender of all groups of children surveyed was represented by a relatively equal percentage of girls and boys. Children of all groups lived in the study areas since their birth and attended educational institutions for children.

Clinical, functional and laboratory examination of the population was carried out according to a specially developed program that takes into account the list of possible risk factors (externally environmental, social, lifestyle, heredity, etc.) and focuses on in-depth examination of the morpho-functional state of organs and systems that are targets for environmental factors.

The procedure for assessing the functional state of the respiratory system was carried out using the spirometry method (Schiller PSspirometry spirometer using the SP260 sensor; Schiller AG, Switzerland) with analysis of lung vital capacity (SVC), forced vital capacity (FVC), forced expiratory volume for the first second (FEV1), Gensler's index (FEV1/FVC), forced expiratory flow at 25, 50 and $75 \%$ of FVC (MEF 25, MEF 50, MEF 75), peak expiratory flow (PEF) and respiratory minute volume ( MV) (to calculate the proper values, the normative standards for children according to Knudson were used), as well as by the rhinomanometry method (rhinomanometry system SRE 2000 with the Rhinostream sensor; Interacoustics A/S, Denmark) with the study of the expiratory flow rate on the left and right, the total flow rate on the left and right, the total total flow rate on both sides. The assessment of the functional reserves of respiratory and cardiovascular systems was carried out by calculating the Skibinskaya index according to the formula: $(0.01 * \mathrm{FVC}) *$ inspiration breath hold $) /$ heart rate, where FVC is forced vital capacity of the lungs $(\mathrm{ml})$; inspiration breath hold is the inspiration breath hold per sec., heart rate is the heart rate (beats per minute). The interpretation was carried out according to the following criteria: less than 5 c.u. - very bad result, 5-10 c.u. - bad result, 10-30 c.u. - average result, 30-60 c.u. - good result, more than 60 c.u. - excellent result.
The laboratory examination included a study of the immune status, i.e. determination of the total content of leukocytes, the relative and absolute content of lymphocytes by standard laboratory methods. The analysis of the relative and absolute number of the following subpopulations of lymphocytes was carried out according to the expression of the corresponding membrane markers: CD4+CD127- activated T-lymphocytes, using the corresponding monoclonal antibodies (BD Biosciences, USA). A suspension of peripheral blood mononuclear cells isolated by centrifugation in a density gradient of ficoll-verografin $\left(\rho=1.077 \mathrm{~g} / \mathrm{cm}^{3}\right)$ (Pharmacia) was used for the analysis. Staining was performed according to the method of the manufacturer of monoclonal antibodies (BD Biosciences, USA). The analysis was performed by flow cytometry on a Becton Dickinson FACSCalibur flow cytometer using the appropriate Becton Dickinson monoclonal antibodies with the help of the CellQuestPrO universal program.

To assess the apoptosis system, the intracellular expression of the protein Bcl-2 and the surface expression of the receptor to the tumor necrosis factor receptor $\alpha 1$ (TNFR1 - tumor necrosis factor receptor) were investigated by flow cytometry using the corresponding monoclonal antibodies (PE Hamster Anti-Mouse Bcl-2 Set (RUO), PE Mouse Anti-Human TNF Clone MAb11 (RUO), BD Biosciences, USA).

Phagocytic activity of cells was studied using formalinized sheep erythrocytes as objects of phagocytosis.

The content of serum immunoglobulins A, G, M was determined by the method of radial immunodiffusion according to Mancini with standard method.

The level of beta2-microglobulin was determined in blood serum by enzyme immunoassay using a commercial kit on an Elx808IU analyzer (BioTek, USA).

The material was processed using Microsoft Office Excel 2003 and Statistica 6.0 for high-quality statistical data analysis (StatSoft, USA). The data were processed by the method of variation statistics with the calculation of the arithmetic mean and its standard error. The significance of the differences was determined by the Student's test, the assessment of the relationship between the signs using correlation and regression analysis, Fisher's test. Qualitative data are presented as absolute or relative $(\%)$ frequencies, quantitative features are presented as $\mathrm{M} \pm \mathrm{m}$ (arithmetic mean \pm mean error). The significance of differences between groups was considered significant at $\mathrm{p} \leq 0.05$.

Modeling of the following dependence "concentration of chemical substances of technogenic origin in blood frequency of registration of a class, group of diseases or a specific nosological form" and "concentration of chemical substances of technogenic origin in blood - frequency of registration of indicators of functional and ultrasonic research methods" was carried out by the method of correlation and regression analysis with verification of statistical hypotheses regarding model parameters. The adequacy of the models was established using one-way analysis of variance. All statistical procedures used a significance level of 0.05 . 


\section{RESULTS}

The analysis of the morbidity structure of children in the group with respiratory diseases showed that in the absence of significant differences the level of prevalence of allergic diseases (ICD 10: J30.3, J39.8, J45.0) in the examined children was 2.4 times higher $(14.2 \%$ is the group of control versus $5.9 \%$, which is the comparison group, $\mathrm{p}=0.10$ ), allergic rhinitis was diagnosed 3.2 times more often $(10.6 \%$ and $3.3 \%$, respectively, $\mathrm{p}=0.22)$. In addition, the children of the control group had bronchial asthma (3.5\%, p=0.30) in the absence of a similar pathology in the children of the comparison group.

More than half of the children $(54.0 \%)$ of the control group had clinical signs of chronic lymphatic proliferative diseases (J35.0, J35.1, J35.2, J35.9), the prevalence of which was significantly higher than in children of the comparison group $2(29.4 \%, \mathrm{p}=0.001)$. Hypertrophy of the palatine tonsils was diagnosed in almost every third examined child in the control group $(39.8 \%)$, while among children in the comparison group, this pathology was found only in $10.0 \%$ of children $(\mathrm{p}=0.001)$. The children of the control group were 3.5 times more likely to have chronic nonspecific inflammatory diseases of the upper respiratory tract (J31.0, J31.2, J31.9), i.e. $61.9 \%$ and $17.6 \%$, respectively $(p=0.001)$, while the inflammatory process was more common: almost $2 / 3$ of children had signs of chronic nasopharyngitis $(61.9 \%$ versus $3.3 \%, \mathrm{p}=0.001$ ), and chronic rhinitis and chronic pharyngitis, as separate nosological forms, were diagnosed 1.6-5.1 times more often than in children of the comparison group 2 (10.6 and $16.8 \%$ is the control group, 6.7 and $3.3 \%$ is the comparison group), however, there were no significant differences in the indicators $(\mathrm{p}=0.06-0.52)$.

The dependence of the frequency of detection in children of chronic nonspecific upper respiratory tract diseases with the concentration of aluminum in urine $(\mathrm{R} 2=0.704 ; \mathrm{p} \leq 0.00)$ was established.
The analysis of the mean group values of spirography indicators in preschool-age children (control group) did not reveal significant differences in the compared groups: mean group indicators of external respiration function corresponded to the physiological norm ( $\mathrm{p}=0.09-0.80)$, but all indicators in children from the control group were 1.2-1.3 times lower than in the children from the comparison group.

A comparative analysis of the registration of variants of the implementation of the external respiration function showed that the normal variant had almost equal number of children in both study groups $(89.2 \%$ in the control group versus $76.9 \%$ in the comparison group, $p=0.2$ ). A comparative study of the frequency of registration of detection of deviations from the norm in spirogram parameters revealed that in the control group as a whole deviations from the norm were 2.1 times less frequent (10.8\% and $23.1 \%$, respectively, $\mathrm{p}=0.2)$; however, the obstructive disorders of mild and moderate severity were recorded only in the control group, but without statistical significance $(\mathrm{p}=0.6-0.7)$.

According to the results of the assessment of rhinomanometry data, $64.4 \%$ of the control group showed a decrease in the patency of the nasal passages, which was mainly mild $(29.7 \%)$. Every fifth in the control group experienced pronounced difficulty in nasal breathing, and only $13.9 \%$ experienced moderate difficulty.

According to the results of this work, in a group of children (observation group) with functional disorders of the respiratory system, there is a significant increase in the level of specific sensitization to aluminum, in relation to the norm and the comparison group by 4.0 and 1.2 times, respectively.

In the course of the study, violations of immunologic resistance were revealed in children living under conditions of technogenic load (Table).

Table 1 Immunity indicators in children with functional respiratory disorders

\begin{tabular}{|c|c|c|c|}
\hline Indicator & Physiological norm & Control group (n=55) & Comparison group (n=38) \\
\hline Bcl-2, \% & $1-1.5$ & $0.795 \pm 0.716^{* *}$ & $2.085 \pm 1.56$ \\
\hline CD4+CD127, rel., \% & $0.8-1,2$ & $4.237 \pm 4.065^{* * * *}$ & $2.548 \pm 1.753$ \\
\hline TNFR1, \% & $1-1.5$ & $2.555 \pm 3.004^{*}$ & $1.008 \pm 0.509$ \\
\hline IgG, g/dm ${ }^{3}$ & $10.96-16$ & $10.287 \pm 0.471^{*}$ & $10.063 \pm 0.494$ \\
\hline Phagoсуtic number, с.u. & $0.8-1,2$ & $0.654 \pm 0.038^{*}$ & $0.62 \pm 0.045$ \\
\hline IgG к алюминию, у.е. & $0-0.1$ & $0.238 \pm 0.051$ & $0.192 \pm 0.065$ \\
\hline бета2-микроглобулин, мкг $/ \mathrm{cm}^{3}$ & $0-0.003$ & $0.901 \pm 0.087$ & $1.05 \pm 0.359$ \\
\hline
\end{tabular}

Note: $*$ - the difference is significant relative to the reference interval $(\mathrm{p}<0.05), * *-$ the difference is significant relative to the comparison group $(\mathrm{p}<0.05)$ 
The children of the observation group living on the territory exposed to aluminum compounds showed a significant increase in the relative number of T-regulatory lymphocytes (Treg) - (CD4+CD127-) in relation to the physiological norm (in $75 \%$ ) and the comparison group by 1.7 times. These results indicate impaired control of the occurrence of neoplasms, the occurrence of lymphoproliferative processes.

The suppression of the expression of the Bcl-2 protein involved in apoptosis was found. In $75 \%$ of children, there is a significant decrease in this indicator in relation to the physiological norm, as well as in relation to the comparison group, i.e. 2.6 -fold decrease $(\mathrm{p}<0.05)$.

The study revealed an increase in the TNFR 1 content relative to the norm (in $75 \%$ of children) and the control group by 2.5 times $(\mathrm{p}<0.05)$. Tumor necrosis factor (TNF, TNF) is considered an important component in killing cancer cells. This protein triggers the body's immune response to any external stimulus. An increase in this indicator may indicate an excessive external activation of immunoregulatory processes, which can lead to the launch of allergic and autoimmune processes, and, as a result, to a possible loss of control over the occurrence of oncological diseases.

In the control group, an increase in the level of beta-2microglobulin was found in $100 \%$ of children in relation to the physiological norm, and in relation to the comparison group, the increase in the level of protein expression was 1.2 times $(\mathrm{p}<0.05)$. Beta-2-microglobulin is a protein found on the surface of virtually all cells in the body. It is present in most physiological fluids and plays an important role in the mechanism of the immune response. Its content in the blood increases in cancer processes such as myeloma, leukemia and lymphoma, as well as in inflammatory conditions. In our case, an increase in the protein beta-2-microglobulin in the blood serum may be associated with excessive haptenic stimulation with aluminum.

\section{CONCLUSION}

The respiratory diseases were diagnosed in children aged 6-11 years living in the area of technogenic chemical factors (observation group) 1.8-1.9 times more often than children in the comparison group. The respiratory diseases in children of the study area are characterized by a 2.4 -fold higher prevalence of allergic diseases, a 1.8-3.5-fold higher prevalence of chronic nonspecific inflammatory diseases of the nasopharynx and processes accompanied by hyperplasia of the lymph-pharyngeal ring associated with aluminum contamination.
According to the results of an immunologic examination of children (control group), a homeostatic imbalance was revealed due to the overproduction of specific $\mathrm{IgG}$ to aluminum, overexpression of the protein beta2-microglobulin, IgG, CD127-, TNFR by 1.2, 1.0, 1.7, 2.5 and 1.2 times, respectively, as well as by inhibition of the $\mathrm{Bcl}-2$ protein by 2.6 times $(\mathrm{p}<0.05)$.

\section{REFERENCES}

[1] V.I. Agarkov, S.V. Grishchenko, V.P. Korovin, Diseases of the circulatory system among the population of the urbanized region, Nord-Press, Donetsk, 2004, 167 p.

[2] V.V. Sumenko, V.M. Boev, S.E. Lebedkova et al., The state of health in children depending on the nature of anthropogenic pollution, Hygiene and Sanitat. 1 (2012) 67-69.

[3] A.S. Bastron, A.V. Zurochka, E.S. Kremleva, Features of the state of the immune system according to comprehensive medical and social immunological monitoring at enterprises of industrial enterprises of the Urals and Siberia, Med. immunol. 7(3) (2005) 216-217.

[4] V.T. Dolgikh, Fundamentals of Immunopathology, Medical Book, Moscow, 2000, 204 p.

[5] V.A. Chereshnev, N.N. Kevorkov, B.A. Bakhmetyev et al., Physiology of the immune system and ecology, Immunol. 3 (2001) 12-16.

[6] O.V. Dolgikh, A.V. Krivtsov, O.A. Bubnova et al., Analysis of indicators of the immune status in children under conditions of aerogenic exposure to metals, Hygiene and sanitat. 96(1) (2017) 26-29.

[7] D.M. MacGillivray, T.R. Kollmann, The role of environmental factors in modulating immune responses in youth, Front. Immunol. 5 (2014) 434.

[8] N.V. Zaitseva, O.Yu. Ustinova, M.A. Zemlyanova, Prevention of respiratory diseases in children under the influence of chemical environmental factors, Hygiene and sanitat. 2 (2014) 23-27.

[9] N.V. Zaitseva, O.Yu. Ustinova, A.I. Aminova, Hygienic aspects of children's health under the influence of chemical environmental factors, Book format, Perm, 2011, 489 p.

[10] V.V. Vasiliev, Contribution of environmental factors to the health of the population of the child population, Occupational med. and human ecol. 3 (2015) 71-74. 